\title{
An experimental study of the alphabetical rating
}

\author{
SERGIO CESARE MASIN, GIULIANA MAZZONI, and GIORGIO VALLORTIGARA \\ University of Padua, Padua, Italy
}

\begin{abstract}
Alphabetical and numerical ratings were compared by requiring observers to rate lightnesses using the letters of the Italian alphabet or integers in the ranges $0-20$ or 1-21. Alphabetical and numerical ratings did not coincide. The hypothesis that the discrepancy depended on a judged difference between interletter and corresponding interinteger distances was tested by asking observers to produce sections of the alphabet. Relatively small letter-search effects were found in the second half of the alphabet. From the results, it seems possible to conclude that interletter and corresponding interinteger distances are equal, and that the discrepancy between the two kinds of ratings might depend on the properties of the output function that transforms internal representations of magnitude into overt responses.
\end{abstract}

A rating method requires the establishment of a correspondence between the elements of a predefined ordered set of labels and a set of sensory values perceived by the observer. The most widely used sets of verbal labels are ordered sets of numerical symbols. Anderson (1982) recommends the set of integers 1-20 for use in the numerical-rating method. Another ordered set is made up of the letters of the alphabet. For example, the Italian alphabet contains 21 letters, the use of which would define an alphabetical-rating method. The range of sensory values perceived by the observer is defined by two sensory end anchors, which usually correspond (by definition) to the two end verbal labels (i.e., 1 and 20 , or A and $\mathrm{Z}$ ).

Textbooks seem to implicitly consider alphabetical and numerical ratings as equivalent (Anderson, 1982, p. 7; Stevens, 1975 , p. 135), yet this is not certain. For example, Stevens and Galanter (1957) showed that adjectival and numerical ratings produced different results under comparable conditions. A comparison of the alphabetical and numerical ratings seems important for another reason. Direct evaluations of distances within numerical sets are prevented by the observer's automatic usage of computing algorithms. Since letters are not used for computations, those hypotheses that prove impossible to be tested directly on numbers should be testable on the alphabet if alphabetical and numerical ratings are equivalent. The first two experiments presented here were designed to check this possible equivalence.

\section{EXPERIMENT 1}

\section{Method \\ Observers. The observers were 28 university students who were asked to participate in the experiment as they entered the psychology depart- ment at the University of Padua.}

S. C. Masin is now at the University of Udine, Udine, Italy. Requests for reprints should be directed to S. C. Masin, Institute of Phylosophy, Pedagogics and Teaching of Modern Languages, Via Antonini 8, 33100 Udine, Italy.
Stimuli. Each of 19 gray squares with side lengths of $22 \mathrm{~mm}$ was placed in the middle of a $120 \times 210 \mathrm{~mm}$ gray rectangle with reflectance of .23 . The reflectance of a square was one of the following: $.02, .04, .06, .08, .11, .13, .16, .19, .23, .26, .31, .35, .40, .46, .52$, $.59, .67, .76$, or .87 . The stimuli, at a distance of $1.5 \mathrm{~m}$ from the observer, were displayed on the frontal-parallel plane by removing a white screen (reflectance .9-.95). The illumination level was constant (about $30 \mathrm{~lx})$.

Procedure. The squares were numbered from 1 to 19 in order of decreasing reflectance. Before the instructions were given, the oddnumbered squares were shown once in the order 1-19.

The experiment consisted of two parts. For half the observers, in the first part (alphabetical rating) we stipulated that the letter A corresponded to the whitest white ever experienced by the observer, and that the letter $\mathrm{Z}$ corresponded to the blackest black ever experienced. The observers were told to assign a letter of the Italian alphabet $(A, B, C, D, E, F$, $\mathrm{G}, \mathrm{H}, \mathrm{I}, \mathrm{L}, \mathrm{M}, \mathrm{N}, \mathrm{O}, \mathrm{P}, \mathrm{Q}, \mathrm{R}, \mathrm{S}, \mathrm{T}, \mathrm{U}, \mathrm{V}$, or Z) to each square in proportion to the lightness of the square. We specified that the closer a dark gray was to the blackest black, the nearer to $\mathrm{Z}$ the letter had to be, and the closer a light gray was to the whitest white, the nearer to $A$ the letter had to be. The subjects could use a given letter as many times as they thought appropriate and were requested to respond as quickly as possible. Corrections of alphabetical responses were allowed. The 19 squares were then presented twice consecutively in a different random order for each observer. The second part of the experiment (numerical rating) was the same as the first part, except that the observer had to use the integers from 0 to 20 . We stipulated that 0 corresponded to the whitest white and 20 to the blackest black ever experienced by the observer. The integers had to be assigned in proportion to the lightnesses of the squares, as observers did when they used the letters of the alphabet.

For the other half of observers, the numerical rating occurred in the first part of the experiment. A session lasted about $20 \mathrm{~min}$.

\section{Results and Discussion}

For computation purposes, the order-position number of each letter and integer was transposed to its corresponding real number in the range $0-20$; that is, $\mathrm{A}-\mathrm{Z}$ and the labels $0-20$ were changed into the real numbers $0-20$. The arithmetic mean for the two real numbers produced by an observer in response to a given stimulus was computed. These single means for each set of two numbers were then used to compute the group arithmetic means for each stimulus.

The results are illustrated in Figure 1a. The reflectance is represented on the abscissa, and the mean rating of light- 

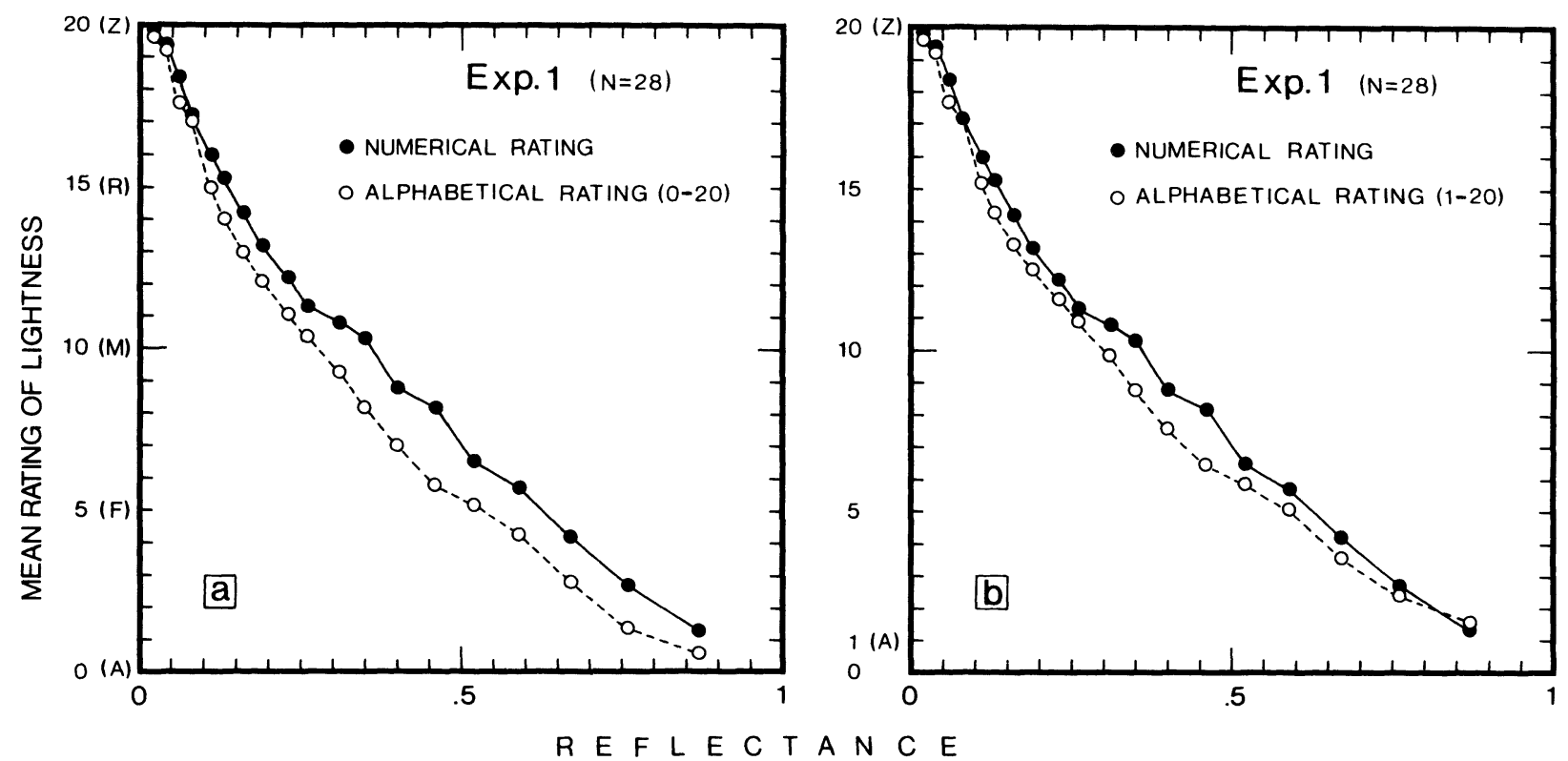

Figure 1. Mlustration of the results of Experiment 1. The graphs show the mean $(N=28$ observers) rating of lightness (ordinate) of gray squares with a given reflectance (abscissa). The ratings were numerical, using integers in the range 0-20 (filled dots), or alphabetical, using the letters of the Italian alphabet (empty dots). On the left (right): results when the letters were transposed to the corresponding number in the range 0-20 (1-20).

ness is represented on the ordinate. The empty (filled) dots show the results of the alphabetical (numerical) rating. The standard errors of the mean for the alphabetical (numerical) ratings ranged from $.13(.07)$ to $.53(.5)$.

A $2 \times 19$ (kind of rating $\times$ reflectance of a square) analysis of variance (ANOVA) was computed. The main effects due to the kind of rating and the interaction of factors were statistically significant $[F(1,27)=31.13$, $p<.00005$, and $F(18,486)=2.16, p<.005$, respectively].

It is commonly believed that there is a close relation between the numerical-rating method and the bisection method (Stevens, 1975, p. 230). Functional-measurement procedures seem to show that the two methods produce the same psychophysical function (Anderson, 1976; Weiss, 1975), suggesting that in both methods the judgmental operations involve representations of sensory distances (Anderson, 1982, p. 134; Carterette \& Anderson, 1979). When the observer rates alphabetically, he/she reasonably retrieves letters from long-term memory and builds up a representational (imagined) alphabet, which he/she scans in search of a letter so that the ratio of the two distances of the letter from the ends of the alphabet equals the ratio of the two corresponding distances of the target sensory value from the ends of the sensory continuum.

It might be that a similar mechanism applies also to integers, and that the label "zero," not being conceived as an integer, was not included in the representational set of integers. ${ }^{1}$ In this case observers should have used the range $1-20$, instead of $0-20$. Consequently, the alphabetical responses should be transposed to a corresponding real number in the range 1-20, instead of the range 0-20. This can be done by first transposing an alphabetical response to the corresponding real number, $n$, in the range $0-20$, and then transforming $n$ into $(19 / 20) n+1$, to make the range 1-20 for letters comparable with the range 0-20 for integers (implicitly equivalent to the range 1-20, since the 0 is not used in the evaluation of distances). Figure $1 b$ illustrates the results after this transformation. An ANOVA indicated that the main effects due to the kind of rating were still significant $[F(1,27)=9.96$, $p<.005]$, whereas the interaction was not significant $[F(18,486)=1.48]$.

The purpose of the next experiment was to determine whether or not observers used the zero. This was done by checking whether the results reported in Figure $1 \mathrm{~b}$ were also obtained when observers rated using the set of integers 1-21.

\section{EXPERIMENT 2}

\section{Method}

Observers. The observers were 28 university students who had not participated in Experiment 1.

Stimuli. The stimuli were the same as in Experiment 1.

Procedure. The procedure was the same as that used in Experiment 1, except that during the numerical rating observers used the integers in the range 1-21. The whitest possible white was defined to correspond to 1 , and the blackest possible black was defined to correspond to 21 . 


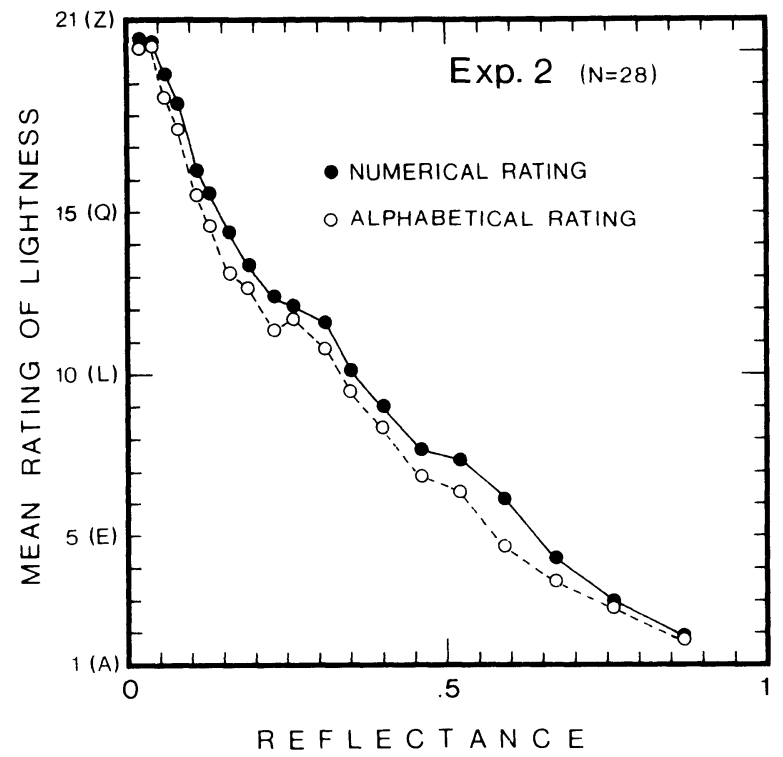

Figure 2. Illustration of the results of Experiment 2, in which observers had to rate numerically (using the range 1-21), or alphabetically (using the range $A-Z$ ). The symbols have the same meaning as those in Figure 1.

\section{Results and Discussion}

The alphabetical responses in the range $\mathrm{A}-\mathrm{Z}$ and the numerical responses in the range 1-21 were changed into the corresponding real numbers $1-21$. The numbers were then processed as in Experiment 1. The results are illustrated in Figure 2, where the symbols have the same meaning as in Figure 1. The standard errors of the mean for the alphabetical (numerical) ratings ranged from .13 (.2) to $.59(.52)$.

An ANOVA indicated that the main effects due to the kind of rating were significant $[F(1,27)=10.16$, $p<.005$ ], but that the interaction was not significant $[F(18,486)=.91]$. These results repeat those depicted in Figure $1 \mathrm{~b}$, which were based on the assumption that observers did not use the zero. The assumption seems therefore to be corroborated.

Results by Banks and Coleman (1981) and growing evidence in favor of numerical-response linearity (Anderson, 1981) suggest that representations of consecutive integers are spaced equal distances apart. If this is so, then the substantial parallelism (lack of interaction) of the two curves in Figure 2 indicates that consecutive letters also were spaced equal distances apart. The two curves were obtained on the implicit assumption that the distance between two given letters and the distance between the two corresponding integers were equal. The upward displacement of the curve for numerical rating suggests either that (1) this implicit assumption was false or (2) the displacement was due to the output function that transformed sensory representations into alphabetical or numerical responses (Birnbaum \& Veit, 1974; Curtis, Attneave, \& Harrington, 1968). The next experiment served to test these two possibilities via the direct sectioning of distances between representational letters.

\section{EXPERIMENT 3}

\section{Method}

Observers. The observers were 30 university students who had not participated in Experiments 1 or 2.

Stimuli. The stimuli, displayed in the middle of a monitor screen, were pairs of integers separated by a question mark. The question mark indicated a letter of the alphabet (sectioning letter). The integer on the left indicated the number of letters in the subset of the alphabet from A to the sectioning letter, including the $\mathrm{A}$ and excluding the sectioning letter. The integer on the right indicated the number of letters in the subset from the sectioning letter to $\mathrm{Z}$, including $\mathrm{Z}$ and excluding the sectioning letter. Therefore, the two integers always totaled 20 . There were 19 pairs of integers, corresponding to the sectioning letters indicated on the abscissa in Figure 3.

Procedure. Observers had to call out the name of the letter corresponding to the question mark. They were told to respond as intuitively or as spontaneously as possible, trying to avoid any sort of counting. They also were told to respond as quickly as possible, but were allowed to correct their responses. The pairs of integers were presented in a different random order for each observer, twice consecutively.

\section{Results and Conclusion}

The results are illustrated in Figure 3. The arithmeticmean actual order-position number of the chosen sectioning letter (i.e., the letter chosen for the question mark) is represented on the abscissa as a function of the actual sectioning letter. The vertical bars indicate the double standard error (computed using as scores the arithmetic mean of the order-position numbers of the two sectioning letters chosen by an observer). The unbroken line indicates the theoretical result in case an observer correctly chose the sectioning letter.

As may be seen, the chosen sectioning letters from the letter $\mathrm{P}$ on are displaced toward the center of the alphabet by about one letter. In selecting a sectioning letter, the

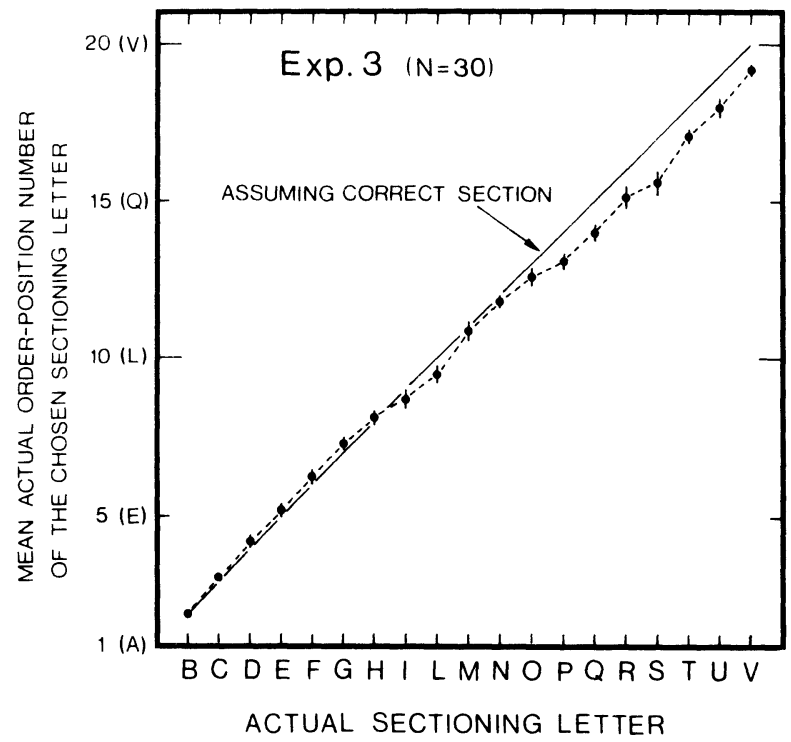

Figure 3. Ilustration of the results of Experiment 3, in which observers had to select a letter of the alphabet given the number of letters on its left and right (sectioning of the alphabet). The mean actual order-position number of the selected letter (ordinate) is plotted as a function of the actual sectioning letter (abscissa). The solid line indicates the theoretical result in case of a correct selection of letters. The vertical bars indicate the double standard error. 
observer had to take into account the entire alphabet. Therefore, since an effect is evident only in the second half of the alphabet (i.e., an asymmetrical effect), observers might have used two different letter-search strategies. Whatever these strategies, however, the displacement of the two curves in Figure 2 also occurred when the sectioning letters (between $\mathrm{A}$ and $\mathrm{O}$; Figure 3) were chosen correctly. If a sectioning letter was chosen in terms of its distances from $A$ and $Z$, relative to the distances of the numbers (on the left and right of the question mark) from 1 to 21, the correct section in portions of the alphabet from $A$ to $O$ indicates that interletter and corresponding interinteger distances were the same, at least in the alphabet portion A-O.

The incorrect sectionings when the letters are in the portion of the alphabet $\mathrm{P}-\mathrm{Z}$ indicate that the sectioning may depend on different letter-search mechanisms activated by the task. Therefore, the utility of the alphabet for those direct tests that are impossible within a set of integers may clearly be limited by letter-search side effects. Since the letter-search side effect is small in magnitude and occurs in only one part of the alphabet, it does not explain the discrepancy between alphabetical and numerical ratings (Figure 2).

Marks (1968) found that a change in numerical range from 1-4 to $1-20$ or to $1-100$ produced a moderate change in the size of the exponent of category scales. By comparison, the letter-search side effect in Experiment 3 consisted of an underestimation of interletter distances of about one letter at maximum, implying a possible change of range from 1-21 to 1-20, which is too small to invoke Marks's effect.

According to Anderson $(1982$, p. 7), the use of word labels in rating is risky because words suggest unequal subjective category widths. If adjectives are changed directly into numbers, then a nonlinear bias would ensue. In fact, Stevens and Galanter (1957) obtained a discrepancy between adjectival and numerical ratings. Our results (Figure 2) showed that a linear bias occurred when word labels were as neutral as letters. The results of Experiment 3, apart from the letter-search side effect, indicated that interletter and corresponding interinteger distances were equal. A reasonable conclusion seems, therefore, to be that the linear bias we found might depend on the properties of the output function that transforms internal representations of magnitude into numerical and alphabetical responses.

\section{REFERENCES}

ANDERSON, N. H. (1976). Integration theory, functional measurement, and the psychophysical law. In H.-G. Geissler \& Y. M. Zabrodin (Eds.), Advances in psychophysics. Berlin, West Germany: VEB Deutscher Verlag.

ANDERSON, N. H. (1981). Foundations of information integration theory. New York: Academic Press.

ANDERSON, N. H. (1982). Methods of information integration theory. New York: Academic Press.

Banks, W. P., \& Coleman, M. J. (1981). Two subjective scales of number. Perception \& Psychophysics, 29, 95-105.

Birnbaum, M. H., \& Veit, C. T. (1974). Scale convergence as a criterion for rescaling: Information integration with difference, ratio, and averaging tasks. Perception \& Psychophysics, 15, 7-15.

Carterette, E. C., \& Anderson, N. H. (1979). Bisection of loudness. Perception \& Psychophysics, 26, 265-280.

Curtis, D. W., Attneave, F., \& Harrington, T. L. (1968). A test of a two-stage model of magnitude estimation. Perception \& Psychophysics, 3, 25-31.

MARKS, L. E. (1968). Stimulus-range, number of categories, and form of the category-scale. American Journal of Psychology, 81, 467-479.

Sartori, G., Roncato, S., Rumiati, R., \& Maso, A. (1986). Reading and processing numbers: Models and neuropsychological investigations. In L. Stegagno (Ed.), Joint Conference of Experimental Psychological Society, Società Italiana di Psicologia (Divisione Ricerca di Base), Società Italiana di Neuropsicologia (Padova, April 2-5, 1986). Milan: Unicopli.

Stevens, S. S. (1975). Psychophysics. New York: Wiley.

STEVENS, S. S., \& GALANTER, E. (1957). Ratio scales and category scales for a dozen perceptual continua. Journal of Experimental Psychology, 54, 377-411.

WEISS, D. J. (1975). Quantifying private events: A functional measurement analysis of equisection. Perception \& Psychophysics, 17, 351-357.

\section{NOTE}

1. This hypothesis seems plausible also in the light of brain lesion studies. Sartori, Roncato, Rumiati, and Maso (1986) described acalculic patients who were able to use the digits but not the zero. This finding suggests that the zero has a special representation in the brain.

(Manuscript received for publication February 16, 1987.) 